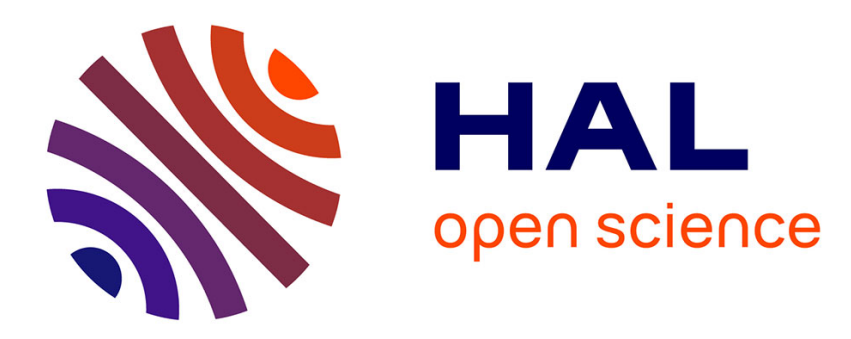

\title{
Gestion publique de l'eau potable, développement urbain durable et majel-s (citernes pour l'eau de pluie) à Sfax (Tunisie)
}

\author{
Ali Bennasr, Éric Verdeil
}

\section{- To cite this version:}

Ali Bennasr, Éric Verdeil. Gestion publique de l'eau potable, développement urbain durable et majel-s (citernes pour l'eau de pluie) à Sfax (Tunisie). Flux - Cahiers scientifiques internationaux Réseaux et territoires, 2009, 76, pp.38-50. 10.3917/flux.076.0038 . halshs-00424458

\section{HAL Id: halshs-00424458 \\ https://shs.hal.science/halshs-00424458}

Submitted on 9 Feb 2016

HAL is a multi-disciplinary open access archive for the deposit and dissemination of scientific research documents, whether they are published or not. The documents may come from teaching and research institutions in France or abroad, or from public or private research centers.
L'archive ouverte pluridisciplinaire HAL, est destinée au dépôt et à la diffusion de documents scientifiques de niveau recherche, publiés ou non, émanant des établissements d'enseignement et de recherche français ou étrangers, des laboratoires publics ou privés. 


\section{CA I R N}

chercher : repérer : avancer

Cet article est disponible en ligne à l'adresse :

http://www.cairn.info/article.php?ID_REVUE=FLUX\&ID_NUMPUBLIE=FLUX_076\&ID_ARTICLE=FLUX_076_0038

Gestion publique de l'eau potable, développement urbain durable et Majels (citernes d'eau pour l'eau de pluie) à Sfax en Tunisie

par Ali BENNASR et Éric VERDEIL

| Métropolis | Flux

2009/2-3 - $\mathbf{N}^{\circ} 76 / 77$

ISSN 1154-2721| pages 38 à 50

Pour citer cet article :

- Bennasr A. et Verdeil n, Gestion publique de l'eau potable, développement urbain durable et Majel-s (citernes d'eau pour I'eau de pluie) à Sfax en Tunisie, Flux 2009/2-3, N 76/77, p. 38-50.

Distribution électronique Cairn pour Métropolis.

(c) Métropolis. Tous droits réservés pour tous pays.

La reproduction ou représentation de cet article, notamment par photocopie, n'est autorisée que dans les limites des conditions générales d'utilisation du site ou, le cas échéant, des conditions générales de la licence souscrite par votre établissement. Toute autre reproduction ou représentation, en tout ou partie, sous quelque forme et de quelque manière que ce soit, est interdite sauf accord préalable et écrit de l'éditeur, en dehors des cas prévus par la législation en vigueur en France. Il est précisé que son stockage dans une base de données est également interdit. 


\title{
Gestion publique de l'eau potable, développement urbain durable et Majel-s (citernes d'eau pour l'eau de pluie) à Sfax en Tunisie
}

\author{
Ali Bennasr \& Éric Verdeil
}

\begin{abstract}
a problématique du développement urbain durable conduit
Là un questionnement sur la réorganisation des services en réseau voire à leur mise en cause au profit de solutions et dispositifs décentralisés, de circuits courts ou de logiques d'autonomie. Ces questions se posent différemment dans les pays du Sud, à forte croissance démographique et urbaine, que dans les pays du Nord. En effet, le fonctionnement des services de base en réseau y apparaît souvent fort insatisfaisant et a d'ailleurs été analysé à travers les notions de non-branchement, de débranchement ou de mal branchement (Jaglin, 2004). Du reste, I'analyse empirique montre bien que se substituent ou s'articulent, de manière complémentaire, d'autres dispositifs de fourniture du service, en général sous normés, par des prestataires alternatifs, souvent informels, et opérant soit hors réseau, soit dans le cadre de mini-réseaux. Ainsi, dans le domaine de l'eau potable en est-il du recours à l'achat d'eau en bouteille, de la collecte des eaux de pluie, ou du branchement à un réseau basique alimenté à partir d'un forage local.
\end{abstract}

Ces systèmes longtemps considérés comme provisoires, dans l'attente d'une mise à niveau et / ou d'une extension du réseau public, s'inscrivent en réalité dans la longue durée, pour ne pas parler de durabilité qui induirait une confusion avec la logique et les préconisations du développement durable, notamment à cause d'une qualité de service inférieure et d'un coût réputé plus onéreux. Quoique, justement, des travaux récents sur l'alimentation en eau potable dans des quartiers de New Delhi en Inde tendent à modifier notre regard. Dans cette ville, les performances du réseau public d'alimentation en eau potable sont notoirement très faibles et n'assurent qu'une desserte intermittente. Dans le cas étudié, une série d'aménage- ments et de dispositifs à l'échelle d'immeubles ou de groupes d'immeubles (habités par des classes moyennes), tels que l'alimentation et le filtrage à partir de forages ou le recueil des eaux de pluie, soutient ou pourrait soutenir, moyennant de petites améliorations et une reconnaissance et une promotion officielles, la comparaison en termes de qualité de service et de coût avec le réseau public (Maria, 2008). Ainsi, la question de I'articulation entre le réseau centralisé et des dispositifs autonomes ou décentralisés, semi-collectifs, paraît-elle pertinente et susceptible de s'intégrer dans une politique de développement urbain durable. Quelles sont les conditions de possibilité d'une telle articulation? La faiblesse des performances du réseau n'est-elle pas, paradoxalement, ce qui permet cette articulation? Inversement, I'application des principes du développement durable ne constitue-t-elle pas un risque pour le réseau?

Nous proposons ici de procéder à l'examen du cas tunisien, et en particulier de dispositifs autonomes observés à Sfax, la deuxième ville du pays, pour nourrir la discussion. Au contraire de nombre de ses voisins immédiats et d'autres pays comparables, les services de base en réseau (eau, électricité et à un degré moindre, assainissement) se distinguent par des performances tout à fait honorables. Fruit d'une implication soutenue des pouvoirs publics dans le long terme, le développement de ces services en réseau traduit leur souci d'une intégration sociale et politique des populations pauvres, en milieu urbain et, aujourd'hui, en milieu rural. Par ce fait même, la diversité des dispositifs alternatifs ou complémentaires destinés à pallier les dysfonctionnements des opérateurs publics est bien moindre en Tunisie qu'ailleurs. 
Par ailleurs, ce bon élève dans la fourniture de services publics fait également figure de pionnier par ses initiatives en faveur de la maîtrise de la consommation d'eau (Treyer, 2002). En effet, confrontée à un climat de type méditerranéen, tendant vers l'aride dans le sud du pays, la Tunisie dispose des ressources en eau parmi les plus faibles au monde. Or, sa croissance démographique, quoique ralentie, est encore vive. Même si l'effort d'économie d'eau porte en premier lieu sur la consommation agricole, la consommation d'eau urbaine fait également l'objet de différentes mesures en vue de sa réduction, à l'initiative de l'opérateur. L'un des instruments privilégiés de cette politique est la politique tarifaire qui doit cependant concilier l'impératif d'économie d'eau - aux effets d'ores et déjà sensibles - à l'impératif social d'une desserte universelle et de qualité, sans remettre en cause les équilibres financiers de I'opérateur public, la SONEDE. On peut faire l'hypothèse que ces évolutions sont prémonitoires de mutations qui affecteront prochainement d'autres grandes villes dans des contextes sociaux similaires à celui de la Tunisie.

Un zoom sur le cas de Sfax met en évidence des pratiques citadines complémentaires de l'approvisionnement par le réseau, notamment le recueil des eaux de pluie dans des réservoirs souterrains appelés localement majels. Ces pratiques sont sinon méconnues, du moins négligées par les autorités alors même qu'elles s'expriment à grande échelle et qu'elles paraissent susceptibles de constituer un complément de la stratégie de développement durable dans le domaine de l'approvisionnement en eau. En examinant ces pratiques, nous réfléchirons sur leur articulation avec le fonctionnement du réseau (1).

\section{LES RÉUSSITES D'UN SYSTÈME PUBLIC DE GESTION DE L'EAU POTABLE}

Le système tunisien d'approvisionnement en eau potable se distingue par une double originalité. À la différence de ces voisins marocains (Miras, Le Tellier, 2005) ou algériens, qui se sont tournés vers des opérateurs privés, en Tunisie c'est une société publique, la Société Nationale d'Exploitation et de Distribution des Eaux (SONEDE), qui continue d'assurer ce service dans les villes et dans une partie de l'espace rural. II est vrai que cette originalité doit être relativisée à l'échelle de l'Afrique, notamment francophone, où de nombreux pays connaissent un fonctionnement similaire (Jaglin, 2005). La deuxième originalité consiste dans les bonnes performances de l'approvisionnement en eau potable, et ce, alors même que, avec des réserves poten- tielles de $460 \mathrm{~m}^{3} /$ habitant/an, la Tunisie fait partie du groupe des pays les plus pauvres en eau dans le monde. En effet, le pays parvient à assurer un accès très large à une eau potable de qualité, tout en assurant la continuité d'un service où les coupures d'eau relèvent de l'exceptionnel.

La décennie 1960 a été marquée en Tunisie par la mise en place de sociétés publiques dans le but de contrôler, de centraliser et d'organiser le secteur des services publics; plusieurs ont vu le jour dont la SONEDE, établissement public à caractère commercial doté de l'autonomie financière. La mission de la SONEDE, née en 1968 (2), consistait en l'exploitation, l'entretien et le renouvellement des installations de captage, de traitement, de transport et de distribution de l'eau sur l'ensemble du territoire national.

Pour réaliser ses objectifs de l'accès pour tous à l'eau potable, la Tunisie a adopté une politique de mobilisation de ses ressources hydrauliques à travers la mise en place de barrages, de lacs collinaires et de forages. Des transferts d'eau sur de longues distances, acheminant les eaux du Nord vers le littoral urbain et touristique ont été réalisés. C'est l'État qui a pris en charge les grandes infrastructures hydrauliques (construction des barrages et bassins de rétention, mise en place des forages profonds...).

\begin{tabular}{|l|c|c|c|c|}
\hline \multicolumn{5}{|c|}{ Tableau 1. Eau potable: } \\
production, vente, nombre d'abonnés \\
\hline Années & 1994 & 1998 & 2002 & 2006 \\
\hline Abonnés (milliers) & 1187 & 1419 & 1706 & 1987 \\
\hline Eau produite (million m ${ }^{3}$ ) & 323,3 & 327,2 & 373,4 & 439,2 \\
\hline Eau vendue en million m $\mathrm{m}^{3}$ & 237 & 255 & 298 & 337,2 \\
\hline Population desservie (milliers) & 6560 & 7201 & 7760 & 10177 \\
\hline $\begin{array}{l}\text { Eau vendue / Abonnés } \\
\text { (m³/hab.) }\end{array}$ & 199,7 & 179,7 & 174,7 & 169,7 \\
\hline $\begin{array}{l}\text { Population / Abonnés (habi- } \\
\text { tants) }\end{array}$ & 5,5 & 5,1 & 4,5 & 5,1 \\
\hline \begin{tabular}{l} 
Source: SONEDE, 2007. \\
\hline
\end{tabular} & & & \\
\hline
\end{tabular}


Depuis I'indépendance en 1956, I'accès à l'eau potable figurait au centre des politiques publiques dans les villes comme dans les campagnes. Les services d'eau et d'électricité ont été parmi les outils majeurs de la légitimation sociale et politique de l'État. En 2006, le taux de raccordement à l'eau potable se monte à $96 \%$ au niveau national, et atteint $100 \%$ en milieu urbain et 90,6 en milieu rural (3). Dans les villes, la politique d'équipement des quartiers non réglementaires en électricité et en eau potable est particulièrement notable.

La politique d'intégration par les services a même, à certains égards, contribué au développement de cet habitat. Ainsi, les acteurs du secteur non réglementaire (propriétaires fonciers et promoteurs) ont commencé à morceler les terrains autour des villes en misant sur l'arrivée des réseaux. Même si le branchement d'eau potable reste tributaire d'une autorisation de bâtir, I'habitat non réglementaire en périphérie bénéficie du raccordement aux réseaux à travers les programmes sociaux et en particulier les projets dits «présidentiels » dont la mise en œuvre est assurée par l'intermédiaire des représentants du pouvoir (gouverneur, délégué du parti) (Legros, 2003). L'intervention de I'acteur public est d'autant plus aisée que les programmes d'action de la SONEDE sont fixés à l'échelon central. Dans I'espace rural, ce sont également des programmes spécifiques qui ont permis l'extension du réseau et l'amélioration du taux de raccordement (Programmes de développement rural, 1973-1984; Programmes de développement rural intégré, 1986-1992; Programmes du Fonds de Solidarité Nationale (FNS 26-26); Programmes Présidentiels).

L'extension très importante du raccordement aux réseaux d'eau potable (et la même chose vaut pour l'électricité) résulte donc d'une combinaison d'un programme volontariste d'intégration des usagers assumé par la SONEDE et bénéficiant de programmes de subventions spécifiques. Cette politique a été suivie à l'encontre des recommandations de la Banque mondiale et sans son soutien après 1985. Elle s'explique par un souci de régulation sociale dans un contexte marqué par des contestations politiques et sociales, d'abord syndicales (1978), populaires (émeutes du pain en 1982) puis islamistes (à partir du milieu des années 1980) (Chabbi, 1999).

Ce développement généreux piloté par l'État n'a pas signifié pour autant un gaspillage des ressources. Il est au contraire allé de pair avec une politique d'économie d'eau (4) touchant tous les secteurs de la consommation: agricole, industriel et domestique. L'opérateur lui-même a entrepris une rigoureuse gestion de son réseau, de lutte contre les fuites. En 2006 (5), le rendement du réseau d'adduction se situe au niveau de $92 \%$ et celui de distribution atteint 84\%. D'autre part, depuis les années 1970, I'usage du goutte à goutte dans l'irrigation a été inculqué aux agriculteurs et largement étendu (Gana, el Amrani, 2006), de même que la réutilisation des eaux usées et traitées dans certaines pratiques culturales (fourrages, terrains de golf...). Les consommateurs domestiques comme les administrations publiques, les lycées, les hôpitaux, les universités, les établissements hôteliers ont été sensibilisés à l'importance de l'économie d'eau. Sur le plan pratique cette sensibilisation s'est traduite par le remplacement des vieilles installations, la mise en place de la robinetterie intelligente, des chasses d'eau économiques dans les toilettes... Des études de la SONEDE ont montré que les gains enregistrés s'élèvent parfois à plus de $40 \%$ du volume de consommation (IME, 2002).

L'extension de l'approvisionnement en eau potable, notamment en ville, constitue un objectif clé du régime qui a à cette fin gardé la mainmise sur la gestion du secteur par un opérateur public centralisé, par d'importantes subventions à la construction des infrastructures et au raccordement de la population. Dans le même temps, la maîtrise de la consommation et la préservation de la ressource constituent, pour un pays pauvre en ressources hydriques, une option stratégique qui a conduit à la mise en œuvre d'une exigeante politique d'économie d'eau. Mais ce système arrive aujourd'hui à un tournant qu'exprime l'évolution de la politique tarifaire.

\section{L'ÉVOLUTION DE LA POLITIQUE TARIFAIRE DE L'EAU POTABLE}

Les réalisations de la SONEDE tiennent pour une part importante aux financements et subventions apportés par l'État tant dans la construction des infrastructures à l'échelle nationale que dans la généralisation du réseau en ville, notamment dans les quartiers populaires. Mais, comme l'exprime le dixième Plan économique et social (2002-2006), I'État tunisien est amené à limiter son interventionnisme dans la sphère économique, afin de réduire son endettement (République tunisienne et alii, 2004). Les entreprises publiques, dont la SONEDE, sont priées d'améliorer leur autofinancement.

Depuis le début des années 1990, la SONEDE a mis en place des mesures visant une plus grande efficacité financière. 


\begin{tabular}{|c|c|c|c|c|c|c|c|c|c|}
\hline \multicolumn{10}{|c|}{ 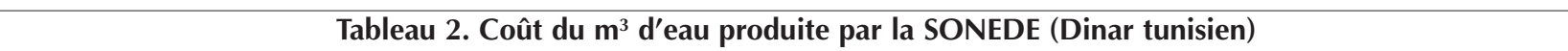 } \\
\hline 1993 & 1994 & 1995 & 1996 & 1997 & 1998 & 1999 & 2000 & 2001 & 2002 \\
\hline 0,379 & 0,370 & 0,382 & 0,460 & 0,434 & 0,462 & 0,462 & 0,471 & 0,477 & 0,505 \\
\hline
\end{tabular}

Ainsi, elle recourt désormais au secteur privé pour certaines opérations techniques qui étaient autrefois de son ressort. Aujourd'hui 94\% des travaux d'extension et 63\% des exécutions des branchements sont sous-traités. De même que plusieurs projets de mise en place de stations de dessalement des eaux de mer sont ouverts à des acteurs privés nationaux et internationaux.

La SONEDE doit cependant faire face à une augmentation structurelle de ses coûts, ce qui implique des évolutions tarifaires. À la phase d'économie d'échelle (1970-2000), matérialisée par l'extension du réseau et l'augmentation du nombre des abonnées en corrélation avec l'évolution de l'urbanisation et des encouragements pour le branchement individuel (6), a succédé une phase de déséconomies d'échelle où les investissements doivent porter de plus en plus sur la réhabilitation des réseaux anciens et sur la recherche de ressources alternatives (dessalement des eaux de mer et des eaux saumâtres). Le taux de croissance du réseau de distribution est passé de 4,5\% par an en moyenne entre 1980 et 2000, à moins de $2 \%$ depuis cette date. Par ailleurs, le coût moyen du $\mathrm{m}^{3}$ ne cesse d'augmenter en raison des nouvelles charges auxquelles la SONEDE doit faire face (tableau 2).

La politique tarifaire de la SONEDE vise à satisfaire trois exigences. D'une part, le tarif doit garantir l'équilibre financier de I'entreprise, ce qui implique des ajustements réguliers pour refléter la hausse des coûts. II a ensuite une finalité sociale. La grille tarifaire comprend classiquement plusieurs tranches de consommation où les tranches supérieures permettent une péréquation entre gros et petits consommateurs. Ainsi, les subventions au secteur domestique proviennent principalement des recettes perçues auprès des usagers industriels et touristiques et des gros consommateurs domestiques (+150 m³/trimestre). Enfin, et plus particulièrement ces dernières années, le tarif a aussi pour finalité l'économie d'eau en dissuadant par un prix élevé les fortes consommations. Ces objectifs étant potentiellement contradictoires, des ajustements réguliers sont nécessaires (Limam, 2007).

La SONEDE a entrepris une nouvelle politique tarifaire dont les principaux objectifs affichés sont l'accès des populations défavorisées à l'eau potable et la sanction des gros consommateurs, en cohérence avec la politique de durabilité de la ressource. Mais ces options risquent de mettre en péril l'équilibre financier de l'entreprise. En effet, les subventions interusages, qui représentent I'un des principes d'équilibre du système, sont de facto menacées par l'évolution des consommations.

La tarification repose depuis 1974 sur un système de tranches et de paliers, auquel s'ajoute une redevance fixe en fonction du diamètre de branchement. Ce système n'a cessé d'être complexifié par la multiplication du nombre de tranches et de paliers, à travers sept étapes (Limam, 2007). Jusqu'en 1981, le palier dit social était situé entre 0 et $40 \mathrm{~m}^{3}$ par trimestre, soit 80 litres/habitant/jour, ramené par la suite à $20 \mathrm{~m}^{3} /$ trimestre. Un second palier de 41 à $70 \mathrm{~m}^{3}$ qui regroupe une majorité de consommateurs, un troisième de 70 à 150 et un dernier de plus de $150 \mathrm{~m}^{3}$ (tableau 3). Les usagers du secteur touristique paient le prix du palier supérieur sur toute leur consommation. Les bornes-fontaines, dont le tarif se situait au niveau de la deuxième tranche à l'origine, sont désormais au niveau tarifaire le plus bas.

La dernière évolution a radicalisé la progressivité du tarif, au nom du reflet nécessaire des coûts de revient, du souci de gérer la demande et de favoriser l'économie d'eau, ainsi que de la volonté de « redressement de la solidarité en vue d'un meilleur ciblage de l'aspect social » (Limam, 2007, p. 5). En clair, depuis 1988, le franchissement du premier palier entraîne l'application du tarif supérieur sur toute la première tranche. La dernière révision a supprimé les paliers à l'intérieur de chaque tranche. Cette tarification pénalise lourdement le dépassement des seuils des différents paliers. Ainsi, un ménage qui consomme $70 \mathrm{~m}^{3}$, paiera 21D (7) (sans compter l'assainissement), un autre ménage qui dépasse ce seuil $\mathrm{d}^{\prime}$ un seul $\mathrm{m}^{3}$ aura sa facture multipliée par 1,85 et paiera 39D.

Du point de vue de l'opérateur, l'intérêt de la réforme est une augmentation des recettes permettant la péréquation avec les petits consommateurs. Les tranches « moyennes » contribuent ainsi davantage à la solidarité. De ce fait, 8,3\% des usagers, consommant $46,6 \%$ de l'eau, assurent $71,9 \%$ des recettes (tableau $\mathrm{n}^{\circ} 4$ ). Ce constat a fait dire à la SONEDE que $90 \%$ des 


\begin{tabular}{|c|c|c|c|c|c|c|c|}
\hline $\begin{array}{c}\text { Paliers de } \\
\text { consommation } \\
\text { en } 1975\end{array}$ & Tarifs en 1975 & $\begin{array}{c}\text { Paliers de } \\
\text { consommation } \\
\text { depuis } 1981\end{array}$ & Tarifs en 1981 & Paliers en 1995 & Tarifs en 1995 & Paliers en 2005 & Tarifs en 2005 \\
\hline \multirow{2}{*}{$0-40$} & \multirow{2}{*}{0,068} & $0-20$ & 0,068 & $0-20$ & 0,115 & $0-20$ & 0,140 \\
\hline & & $21-40$ & 0,075 & $0-40$ & 0,170 & $0-40$ & 0,240 \\
\hline \multirow{3}{*}{ Plus de 40} & \multirow{3}{*}{0,09} & $41-70$ & 0,110 & $41-70$ & 0,338 & $0-70$ & 0,300 \\
\hline & & \multirow{2}{*}{$71 \mathrm{et}+$} & \multirow{2}{*}{0,150} & $71-150$ & 0,525 & $0-150$ & 0,540 \\
\hline & & & & Plus de 150 & 0,650 & Plus de 150 & 0,840 \\
\hline
\end{tabular}

\begin{tabular}{|c|c|c|c|c|c|c|}
\hline \multicolumn{7}{|c|}{ Tableau 4. Structure des abonnés, de la consommation et des recettes de la SONEDE } \\
\hline \multirow{2}{*}{$\begin{array}{l}\text { Tranche } \mathrm{m}^{3} \\
\text { par trimestre }\end{array}$} & \multicolumn{2}{|c|}{ \% des abonnés } & \multicolumn{2}{|c|}{$\%$ de la consommation } & \multicolumn{2}{|c|}{$\%$ des recettes } \\
\hline & 1984 & 1990 & 1984 & 1990 & 1984 & 1990 \\
\hline $0-20$ & 39,5 & 41,1 & 6,3 & 9,1 & 2,0 & 2,7 \\
\hline $21-40$ & 29,3 & 31,3 & 13,1 & 21,7 & 4,6 & 11,1 \\
\hline $41-70$ & 18,1 & 19,4 & 14,5 & 22,6 & 7,1 & 14,3 \\
\hline $71-150$ & 9,8 & 6,8 & 14,5 & 14,6 & 11,8 & 17,0 \\
\hline$>150$ & 3,3 & 1,5 & 51,6 & 32,0 & 74,5 & 54,9 \\
\hline Ensemble & 100 & 100 & 100 & 100 & 100 & 100 \\
\hline
\end{tabular}

consommateurs paient l'eau en dessous de son prix de revient (8). La réforme tarifaire incite tous les consommateurs à ajuster leur consommation pour éviter de tomber dans le palier supérieur plus onéreux.

Cette réforme suscite de la part des consommateurs une certaine insatisfaction qui, comme souvent en Tunisie, ne peut s'exprimer ouvertement. Une chronique de presse récente s'en fait pourtant l'écho, d'autant plus significatif (9).

\section{UNE BAISSE DE LA CONSOMMATION UNITAIRE}

L'application des mesures liées à la gestion de la demande et à l'économie d'eau, et en particulier les conséquences de l'évolution de la politique tarifaire, se traduit par une diminution de

\begin{tabular}{|l|c|c|c|}
\hline \multicolumn{5}{|c|}{ Tableau 5. Évolution des consommations unitaires } \\
\hline Consommations moyennes \Année & 1990 & 2005 & $\begin{array}{c}\% \text { d'évolution } \\
\text { annuelle }\end{array}$ \\
\hline Domestique (m³/an/abonné) & 137 & 125 & $-0,6 \%$ \\
\hline Collectif (m³/an/abonné) & 1300 & 520 & $-5,9 \%$ \\
\hline Hôtellerie (Litre/jour/lit) & 610 & 490 & $-1,5 \%$ \\
\hline Tout usage (m³/an/abonné) & 207 & 170 & $-1,3 \%$ \\
\hline Source: SONEDE. Tableau repris de Limam 2007, p.7. \\
\hline
\end{tabular}

la consommation d'eau des ménages (tableau 5). Les données globales montrent un ralentissement de la croissance de la consommation des abonnés, de 1,3\% en moyenne entre 1990 et 2005 .

La réduction la plus sensible concerne les abonnés collectifs (bâtiments et établissements publics), signe de l'implication des institutions étatiques dans la politique d'économie d'eau ainsi que de l'incitation financière du tarif.

S'agissant des abonnés domestiques, deux effets contradictoires doivent être pris en compte. D'un côté, la politique de branchement universel à l'eau potable (cf. infra) s'est traduite par une hausse de la consommation. De l'autre, la généralisation du branchement individuel a contribué au ralentissement de l'évolution de la consommation. Dans les années 1980, la SONEDE a entrepris une vaste campagne d'incitation aux branchements individuels au détriment des branchements collectifs qui étaient prédominants dans les immeubles et les étages de maison destinés à la location et ce, à travers le paiement échelonné des prix du compteur sur des périodes allant jusqu'à huit ans. Le branchement individuel s'est 


\section{ENCADRÉ 1. BAISSE DES CONSOMMATIONS UNITAIRES D'EAU POTABLE DANS LES PAYS DU SUD: LA TUNISIE À L'AVANT-GARDE?}

Les données de la littérature manquent cruellement pour comparer la situation tunisienne à celle d'autres pays. Rappelons que depuis les années 1990, de nombreux pays européens connaissent une baisse des consommations unitaires. À Paris, par exemple, la consommation a baissé de 2,2\% en moyenne entre 1991 et 1998 (IME, 2002). Ces évolutions touchent surtout les gros consommateurs, mais pas uniquement. Elles sont attribuables à l'usage de nouvelles technologies moins consommatrices, à l'individualisation des compteurs, à la hausse des prix volumiques et à la diffusion d'une sensibilisation aux économies d'eau.

Certaines indications tendent à suggérer que des pays comparables à la Tunisie du point de vue du niveau de richesse suivent une tendance similaire. Ainsi, au Maroc, plusieurs auteurs signalent une tendance à la baisse de la croissance de la consommation d'eau dans les grandes villes, voire une stagnation (Miras, Le Tellier, 2005; Tenneson, Rojat, 2003). Selon Haggi (2006), la dotation brute en milieu urbain à l'échelle nationale est passée de 170 l/hab/j en 1990 à 144 en 1999. Toutefois, les chiffres moyens masquent des tendances divergentes. En effet, l'augmentation des raccordements notamment de ménages pauvres signifie que ces ménages ont de faibles consommations. Inversement, les classes moyennes qui achètent des équipements consommateurs ont des consommations en croissance. La Tunisie, où la généralisation du raccordement est achevée en ville et en bonne voie dans les campagnes, se situe dans une situation intermédiaire.

avéré un outil efficace contre le gaspillage de l'eau jadis observé dans l'habitat collectif suite aux fuites d'eau non réparées. Au total, on constate sur les quinze dernières années une consommation unitaire en diminution régulière $(-0,6 \%$ par an).

Les baisses de consommation constatées pour les autres catégories d'abonnés résultent pour leur part principalement d'une adaptation à l'évolution de la politique tarifaire. Ainsi, le secteur hôtelier a une consommation en baisse de 1,5\% par an entre 1990 et 2005. Les études sur l'élasticité de la demande entreprises par la SONEDE ont montré que le palier supérieur (+ de $150 \mathrm{~m}^{3} /$ trimestre) qui regroupe les gros consommateurs domestiques est le plus élastique (IME, 2002; Limam, 2007). L'effet de la politique tarifaire suivie est d'inciter les plus gros consommateurs à réduire leur consommation. De fait, le tableau 4 montre que la part des plus gros consommateurs a très fortement diminué, passant entre 1990 et 2005 de 51,6\% du volume consommé pour $3,3 \%$ des consommateurs à $32 \%$ pour $1,5 \%$.

C'est dans cette catégorie d'usagers qu'on trouve les industriels. Selon plusieurs observateurs, nombre d'industriels ont adopté des stratégies visant d'une part à ajuster leur consommation d'eau mais aussi à se tourner vers des sources non conventionnelles, telles que des forages, des puits, voire la réutilisation des eaux usées (Limam, 2007). Ces tendances sont aussi visibles à Sfax, où les industries consommatrices d'eau ont de plus en plus recours à ces moyens.

\section{LES ENJEUX ÉCONOMIQUES DE LA BAISSE DES CONSOMMATIONS UNITAIRES}

La SONEDE se trouve aujourd'hui dans une situation de transition. Jusqu'à récemment, cette entreprise faisait face au défi de la croissance mais elle avait réussi, mieux que dans beaucoup d'autres pays, à tirer parti des bénéfices classiquement associés à la croissance: hausse du nombre d'abonnés, hausse des volumes consommés par les usagers, hausse des recettes. Aujourd'hui, la baisse des consommations unitaires, en partie sous l'effet d'une contrainte extérieure qui est la politique d'économie d'eau, enjeu stratégique pour l'État tunisien, représente l'amorce d'une nouvelle phase dont il faut envisager les effets potentiels.

L'équilibre économique de l'exploitation n'est actuellement pas mis en péril, selon les informations indirectes en notre possession. Au contraire d'autres entreprises publiques tunisiennes dont les rapports d'activités et les données financières de base sont disponibles en ligne, la SONEDE ne publie pas ces informations et elles ne nous ont pas été communiquées. Selon un rapport récent co-publié par la Banque mondiale et le ministère tunisien de la coopération internationale, il apparaît toutefois que la SONEDE présente un bilan positif (République tunisienne et alii, 2004) (10).

Cet équilibre repose largement sur une péréquation entre tranches tarifaires dont nous avons précédemment présenté le 
principe. Or, la politique tarifaire pénalisant les gros consommateurs a érodé ce mécanisme de subventions des usagers sociaux par les gros consommateurs, au fur et à mesure que ces derniers ont réduit leurs consommations ou se sont tournés vers des ressources non conventionnelles. C'est d'ailleurs cette évolution qui a rendu nécessaire la dernière réforme tarifaire.

L'équilibre financier de l'entreprise repose également sur une autre péréquation, entre l'espace urbain et l'espace rural. Selon Limam (2008), I'État tunisien a en effet effectué un important effort d'approvisionnement en eau potable des zones rurales depuis le VIII ème plan (1987-91) et, en 2007, seulement $8 \%$ de la population rurale $n^{\prime}$ est pas raccordée. Comme en ville, une politique incitative aux branchements individuels porte ses fruits. La SONEDE est progressivement chargée de la gestion et de l'exploitation de ces zones. Toutefois, en raison des coûts d'exploitation plus élevés et d'une consommation qui reste basse (en moyenne, $60 \mathrm{l} / \mathrm{j} / \mathrm{h}$ habitant), le taux de couverture du coût de l'eau demeure très faible à $54 \%$; alors qu'en ville, il se situe en 2007 à 110\% (Limam, 2008, pp. 11-12).

La politique d'inclusion sociale (y compris la généralisation

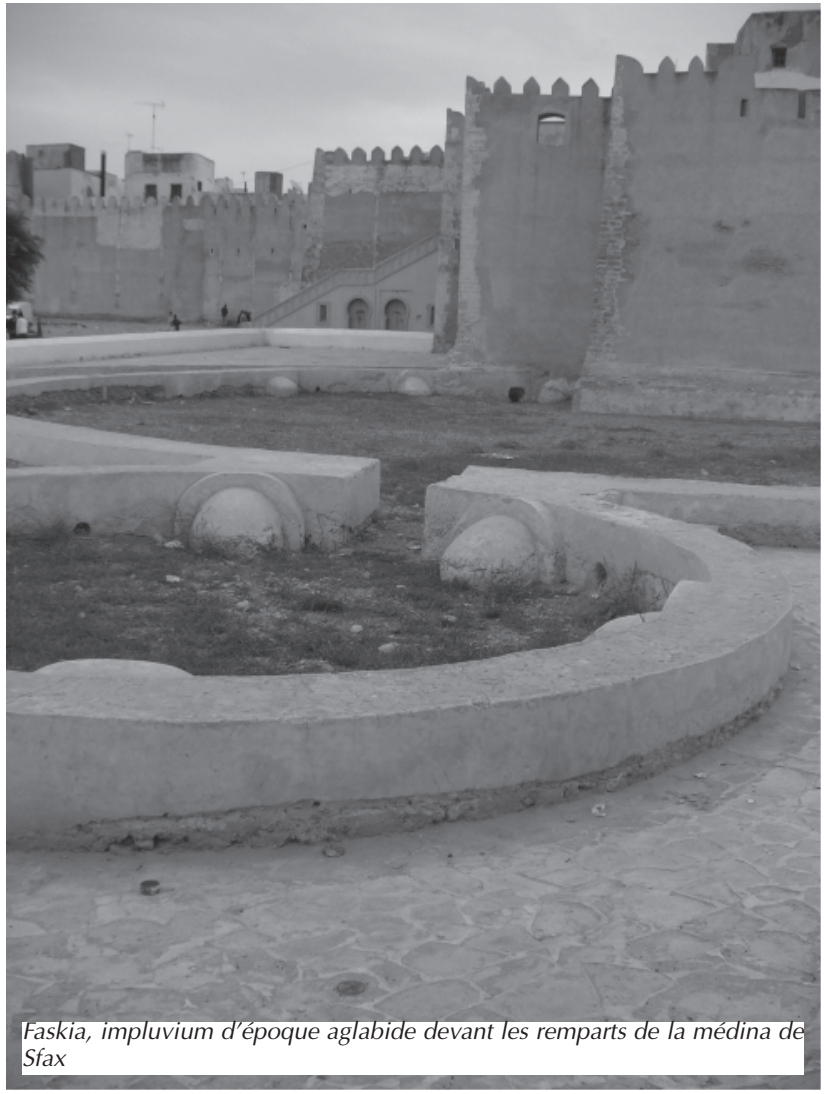

des branchements individuels) et territoriale dont la SONEDE est l'exécutant et sa politique de découragement de gros consommateurs se traduisent donc par une diminution tendancielle des recettes moyennes, dans un contexte où les charges d'exploitation ont plutôt tendance à augmenter. Cette situation n'est apparemment pas encore dommageable pour l'équilibre des comptes, mais elle pose question pour l'avenir.

La réponse tarifaire de nombreux opérateurs d'eau potable dans les pays du Nord confrontés à une décroissance des consommations a notamment consisté en une augmentation des prix (part fixe ou prix volumique). Cependant, cette politique semble être exclue pour l'instant par la SONEDE qui met en avant qu'elle pèserait avant tout sur les usagers les plus démunis et qu'elle serait donc socialement injuste (Limam, 2007 , p. 5).

C'est dans ce contexte des conséquences de la baisse des consommations sur les finances futures de la SONEDE et des réponses incertaines qui lui seront apportées que I'on doit essayer de mieux apprécier le développement des pratiques hors réseau.

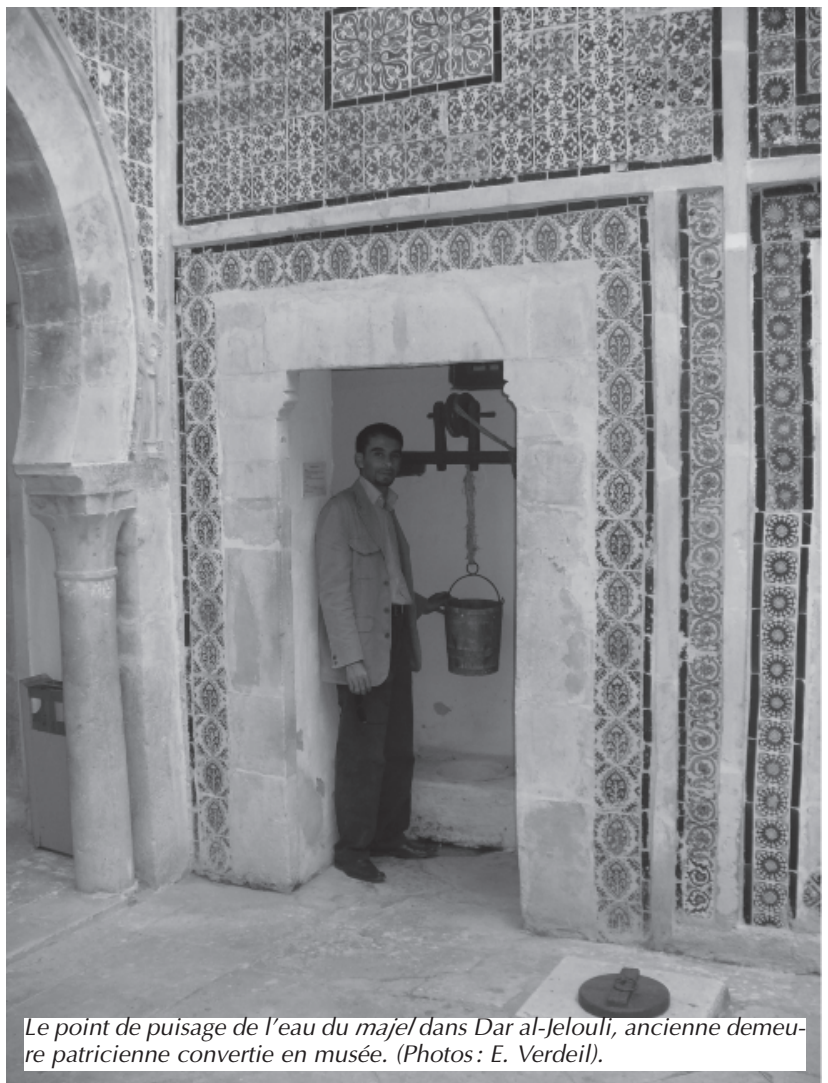




\section{LE DÉVELOPPEMENT DES PRATIQUES HORS RÉSEAU EN QUESTION}

Analyser le développement des pratiques d'approvisionnement hors réseau revient à explorer un paradoxe. En un sens, ces pratiques sont la conséquence d'une mesure prise, entre autres justifications, au nom du développement durable, en l'occurrence la limitation de la pression sur la ressource, l'injonction aux économies d'eau. Mais ces pratiques semblent également porteuses d'une menace, à terme, sur l'équilibre économique de l'approvisionnement en eau potable, et d'autre part, leur développement hors du contrôle étatique est lourde de menace environnementale et sanitaire, et elles seraient donc, de ce point de vue, en contradiction avec l'objectif initial.

Une première modalité de ces pratiques hors réseau dans le cas tunisien concerne le débranchement partiel des gros consommateurs du réseau d'approvisionnement de la SONE$\mathrm{DE}$, en réaction à l'augmentation des tarifs. Nombre d'entre eux semblent se tourner vers des ressources non conventionnelles telles que des forages ou des puits, ou le recyclage des eaux usées pour l'arrosage (11). À vrai dire, ce phénomène est impossible à chiffrer avec précision dans l'état actuel de nos informations bien qu'il soit mentionné par des représentants de la SONEDE (Limam, 2007).

Dans le cas de Sfax, le deuxième centre industriel du pays, les consommations à usage industriel qui accaparaient $12 \%$ du volume de consommation en 1980 ne représentent plus que $9 \%$ du total en 2005. Pourtant, la ville a non seulement conservé son infrastructure industrielle, mais elle l'a enrichie dans la décennie 1980-1990, faisant passer le nombre des entreprises de plus de 10 emplois de 340 unités en 1980 à 660 en 2005 auxquelles s'ajoutent plus de 300 huileries. Les industries utilisatrices de l'eau comme entrant ou dans les circuits de refroidissement ont même gagné du terrain: huileries, industries agroalimentaires (minoteries, pâtes alimentaires...), industries chimiques, marbreries... (Bennasr, 1993, 2004).

Toutefois, de nombreux entretiens sur le terrain ont montré qu'une part non négligeable des industriels a quitté le réseau, même s'il est délicat de fournir une estimation. Depuis longtemps, plusieurs usines possédaient leur propre système d'eau: c'est le cas de l'usine SIAPE, une grande unité de traitement des phosphates créée en 1952 et qui puise l'eau d'un forage profond qu'elle a réalisé. De la même manière, plusieurs usines utilisant l'eau pour le refroidissement (marbreries, industries mécaniques, céramiques...) possèdent leur propre système de recyclage qui fonctionne en mode fermé où l'eau utilisée, puisée de la nappe, est filtrée et réutilisée. Ces observations paraissent cohérentes avec la diminution relative de la consommation industrielle mais devraient être confirmées par des enquêtes quantitatives.

Une conséquence de ces pratiques est en revanche avérée. Le pompage superficiel de la nappe (qui ne nécessite pas d'autorisation administrative), qui fait éviter aux industriels les factures de la SONEDE, constitue une menace écologique car les eaux traitées sont parfois réinjectées dans la nappe. Ces observations menées à Sfax sont très probablement généralisables à l'échelle du pays. Le développement des pratiques hors réseau des gros consommateurs, notamment industriels, doit donc s'analyser comme un effet pervers de l'augmentation régulière des tarifs en raison de ses conséquences contraires à la gestion durable de la ressource en eau.

Un deuxième type d'approvisionnement en eau hors réseau concerne les majel-s, des réservoirs individuels de collecte des eaux de pluie.

\section{LES MAJEL-S, UNE PRATIQUE DURABLE MAIS NÉGLIGÉE: LES PARADOXES D'UN DISPOSITIF HORS RÉSEAU À SFAX}

Sfax, deuxième centre urbain du pays, ville littorale du centreest, un demi-million d'habitants et 1 million dans le gouvernorat, présente l'exemple de systèmes d'approvisionnement en eau potable hors réseau. Ces pratiques, inscrites dans I'histoire, prennent une nouvelle signification et une importance dans le contexte de transformation de la distribution de l'eau potable en Tunisie. La ville, située en limite du climat aride (précipitations d'environ $200 \mathrm{~mm}$ ) était depuis le début du vingtième siècle soumise à un grave problème de pénurie d'eau. Historiquement, une part non négligeable des ressources hydrauliques qui alimentaient la ville provenait des citernes particulières nommées majel-s que possédaient chaque maison et chaque édifice public. Pratique ancestrale, le majel consiste en un gros réservoir creusé au pied des maisons ou sous elles pour la collecte des eaux de pluie sur les toits terrasses. Il y avait en outre en dehors des remparts deux immenses réservoirs appelés faskia, qui viennent d'être exhumés et qui remontent à l'époque aghlabide (XII siècle); et plus près des murs, une vaste enceinte murée, désignée sous le nom de nasria (12), contenait plu- 
sieurs centaines de citernes distinctes, fondées et entretenues par des legs pieux. Certains historiens disent qu'elles égalaient en nombre celui des jours de l'année. Le système de réservoirs a continué à fonctionner jusqu'au milieu du XXe siècle.

Avec le développement urbain que la ville a connu sous la colonisation (mise en place du quartier colonial, extension sur la périphérie), les ressources mobilisées ne suffisaient plus aux besoins. Ainsi, depuis 1910, une nouvelle adduction a été mise en place, engendrant le début d'un transfert de l'eau sur de grandes distances. La ville de Sfax est désormais alimentée par un réseau d'adduction qui achemine les eaux de la source de Sbeïtla et des forages situés à $180 \mathrm{~km}$ à l'ouest de la ville. En 1978, une nouvelle adduction a été posée; elle véhicule les eaux de 21 forages captant les nappes du centre ouest du pays. Enfin, pour répondre à la demande sans cesse croissante, une nouvelle adduction conduisant les eaux du nord du pays a été posée et donne un appoint pour Sfax.

Le volume d'eau distribué par la SONEDE dans le Grand Sfax est passé de 11,16 millions de $\mathrm{m}^{3}$ en 1981 à $30,8 \mathrm{Mm}^{3}$ en 2005 (tableau 6); soit une multiplication par trois, alors que dans le même temps, la population a doublé passant de 240000 à 500000 habitants. On retrouve donc à Sfax la tendance nationale à la baisse de la consommation unitaire même si la ville est connue pour avoir le plus haut niveau de consommation domestique du pays, en particulier en raison des pratiques d'arrosage des jardins (jnaïn-s) avec l'eau du réseau public (Treyer, 2002, annexe 4).

Le développement de l'approvisionnement en eau potable par le réseau public, qui dessert à Sfax comme ailleurs dans les villes tunisiennes, pratiquement tous les logements $(98 \%$ en

Tableau 6. Évolution de la consommation d'eau potable fournie par la SONEDE dans le Grand Sfax $\left(\right.$ en $\left.^{3}\right)$

\begin{tabular}{|l|c|c|c|c|c|}
\hline Année & $\begin{array}{c}\text { Domestique } \\
\text { individuel }\end{array}$ & Collectif & Industrie & Divers & Total \\
\hline 1980 & 7134 & 2118 & 1360 & 550 & 11162 \\
\hline 1985 & 11607 & 1774 & 567 & 598 & 14546 \\
\hline 1990 & 12397 & 2683 & 1245 & 429 & 16754 \\
\hline 1995 & 15813 & 2205 & 1656 & 363 & 20037 \\
\hline 2000 & 19733 & 2941 & 2309 & 503 & 26487 \\
\hline 2005 & 24181 & 3265 & 2754 & 640 & 30840 \\
\hline 2006 & 24401 & 3187 & 2784 & 420 & 30792 \\
\hline \multicolumn{5}{|l}{ Source: SONEDE Sfax } \\
\hline
\end{tabular}

2004), a conduit à une marginalisation partielle des anciennes pratiques de collecte des eaux de pluie. En particulier, les anciens systèmes de citerne collective ont été délaissés. En revanche, ces pratiques persistent parmi les habitants, non seulement dans la ville ancienne (médina) où les dispositifs sont toujours entretenus, mais également dans de nombreuses constructions modernes à la périphérie.

Creusé sous les maisons ou dans les jardins, le majel est une construction en dur, où les eaux de pluie recueillies sur le toit terrasse sont collectées grâce à une gouttière. On peut distinguer deux pratiques. Le majel proprement dit a une forme conique, sa profondeur varie de 4 à 8 mètres sur un diamètre qui atteint jusqu'à $4 \mathrm{~m}$. La feskia est moins profonde que le majel (3 à 4 mètres), et est construite lorsque la croûte calcaire est compacte (difficulté de creuser) ou lorsque la nappe n'est pas profonde (risque d'effondrement). Toutefois, les gens préfèrent le majel, dans la mesure où il prend moins d'emprise et où l'eau est plus fraîche. Le majel est entretenu par la chaux appliquée tous les ans sur les toitures et par l'eau de javel. L'usage traditionnel de l'extraction de l'eau par le seau permet d'oxygéner cette dernière en luttant contre la stagnation (figure 3). Aujourd'hui, sédentarité oblige, de plus en plus de majel-s sont équipés de moteurs électriques, ce qui porte préjudice à la qualité des eaux.

Il n'est pas aisé de donner l'effectif des majel-s dans le Grand Sfax. II paraît acquis que la quasi-totalité des maisons pavillonnaires et des constructions individuelles dans les jardins (jnaïn-s) en sont équipées. Ces types d'habitat (pavillonnaire et dans les jnaïn-s) constituent un tissu urbain en développement; leur part est passée à 50\% de l'ensemble des logements du Grand Sfax (Bennasr, 2003). Les études prospectives ont montré que cette part serait de 70\% à l'horizon 2016 (SDGS, 2003-2006). Or, au recensement de 2004, le Grand Sfax comptait 73000 villas et 52000 logements horizontaux à patio (dar) sur un total de 150000 logements. Mais il est certain qu'il y a autant de majel-s que de maisons pavillonnaires et de maisons à patio dont celles de la Médina. Même dans les quartiers informels, le majel occupe une place de choix, dans la mesure où il permet de sécuriser la ressource en attendant l'arrivée du réseau. II joue aussi exceptionnellement le rôle de réservoir 


\section{ENCADRÉ 2. DeuX ENJEUX À PRENDRE en COMPTE POUR LE DÉVELOPPEMENT DES MAJEL-S}

Le premier enjeu est celui de l'économie d'eau, dans un pays où la ressource en eau est limitée et son usage très contraint. À cet égard, le système du majel constitue sans nul doute un potentiel à encourager. Mais pour disposer d'une image plus claire, un recensement du nombre de logements concernés, de leur capacité et de leur état, serait un préalable à une éventuelle politique de réhabilitation ou d'entretien. Une politique d'encouragement à la construction de nouveaux majel-s ou d'autres types de citernes, adaptés à l'habitat collectif voire, comme le suggère Treyer (2002), des réservoirs collectifs, pourrait être envisagée.

Le deuxième enjeu est sanitaire. La préférence citadine pour l'eau du majel, si elle est avérée par les enquêtes, devrait être mise à l'épreuve d'une surveillance. Il existe pourtant le réseau de laboratoires d'analyses de la SONEDE et celui du ministère de l'Agriculture. Ces derniers, présents dans les régions rurales, offrent leurs services gratuitement. Les analyses portent plus sur la bactériologie, la teneur en sel que sur les métaux lourds, véritable danger pour la ressource. Pourtant, les propriétaires ne font que rarement des analyses bactériologiques de l'eau. À cet égard, une politique de sensibilisation aux enjeux sanitaires et d'entretien paraît nécessaire. Outre le risque bactériologique, la pollution urbaine présente un risque surtout dans les zones proches du centre-ville et des zones industrielles: les métaux lourds dégagés des échappements de véhicules et des cheminées de certaines industries qui se précipitent dans les réservoirs ne sont pas à négliger.

pendant les périodes de sécheresse où il est rempli via des citernes par l'eau des puits ou de la SONEDE. Si on considère que $90 \%$ des villas renferment un majel et que $3 / 4$ des dar en possédaient, on arrive à environ 104000 majel-s. Si, en moyenne, la contenance d'un majel est d'environ $35 \mathrm{~m}^{3}$ (estimation d'après un échantillon aléatoire), on obtient ainsi un volume de 3,5 millions de $\mathrm{m}^{3}$ soit $14 \%$ du volume d'eau distribué par la SONEDE et consommé pour les usages domestiques. Cette estimation est supérieure au chiffre de 4,5\% proposé par Treyer (2002, annexe 4, p. 27) avec laquelle la comparaison est difficile, faute $d^{\prime}$ indications méthodologiques et d'un espace de référence plus large (gouvernorat). On doit la considérer comme un ordre de grandeur.

Malgré les incertitudes qui entourent son estimation, la collecte de l'eau de pluie dans les majel-s correspond bien à un usage vivant et même réactivé dans la ville de Sfax. Pratique ancestrale telle qu'attestée par les vestiges archéologiques (romains

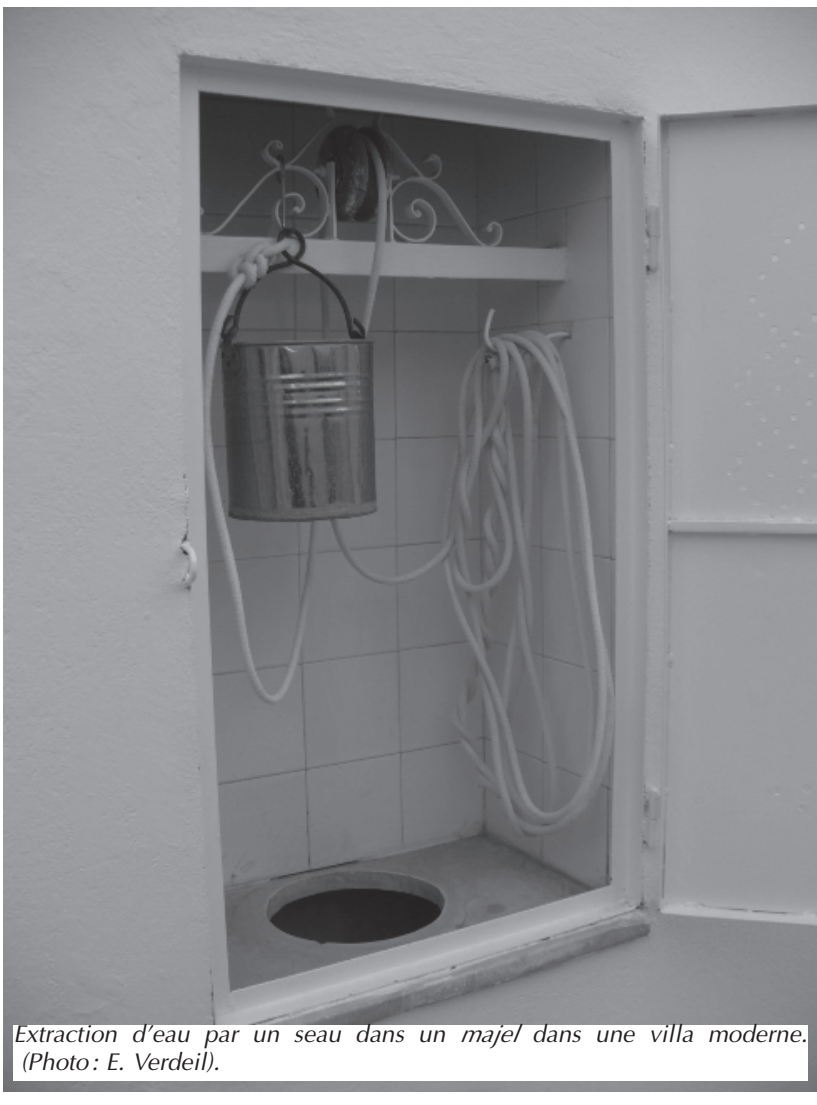

et arabo-musulmans), le majel est entré dans les mœurs et dans la culture des populations de la région de Sfax, à la ville comme à la campagne. La tradition veut que creuser un majel pour offrir de l'eau aux demandeurs équivaut à un pèlerinage à La Mecque. La culture de la rareté fait que les gens dans les campagnes mais aussi en ville ont gardé I'habitude de creuser des majel-s qui permettent de sécuriser les ressources en eau.

Mais la raison principale du recours au maje/ vient de la préférence des habitants pour cette eau du réservoir. Lorsque les logements en sont pourvus, leurs habitants préfèrent en effet utiliser l'eau du majel pour les usages alimentaires et la boisson. En dépit de sa faible teneur en sels minéraux, l'eau du majel présente un goût nettement meilleur que celle du robinet caractérisée par une salinité plus élevée et des résidus calcaires. Or, si les précautions d'entretien sont bien respectées, elle ne présente aucun danger bactériologique pour la santé. De manière 
paradoxale, les Sfaxiens consomment donc l'eau gratuite du majel et utilisent massivement celle du réseau pour l'arrosage (Treyer, 2002) et d'autres usages (hygiène, nettoyage). En effet, l'eau puisée dans la nappe, désormais très salée, n'est pas de qualité suffisante pour cet usage a priori non noble.

Les majel-s représentent donc l'exemple d'une pratique conforme au principe de l'économie d'eau coexistant avec des pratiques qui s'apparentent à un gaspillage économique: I'utilisation de l'eau potable pour le jardinage. Ces pratiques paradoxales constituent un défi en termes de politique publique et d'économie.

Le système des majel-s constitue essentiellement une spécificité sfaxienne, qu'on ne retrouve à grande échelle que dans deux autres villes tunisiennes: Mahdia et Djerba. Sans doute pour cette raison, le potentiel des citernes comme sources d'approvisionnement en eau potable à l'échelle de la Tunisie ne semble pas évoqué dans les documents officiels de planification des eaux, ni dans l'Atlas de l'eau en Tunisie (Hénia, 2008). Si l'analyse prospective de l'eau réalisée par le Plan bleu (Treyer, 2002) mentionne les citernes, ce n'est que dans une annexe, à la faveur d'un scénario d'évolution à l'échelle régionale de Sfax (Treyer, 2002, annexe 4) (13). Toutefois, il est juste de signaler que si le gouvernement tunisien ne prend pas en considération les citernes dans la panoplie des sources d'approvisionnement dans les villes, il encourage en revanche leur construction dans l'espace rural (14).

Il est difficile, faute d'entretiens menés systématiquement sur ce point avec les responsables de la SONEDE à différents échelons de l'administration, d'apporter une explication définitive à cette situation qui, vue de Sfax, paraît globalement étonnante eu égard à l'intérêt potentiel des majel-s dans le contexte de l'économie d'eau. On se contentera ici de deux hypothèses complémentaires. Précisons aussi que la mise en avant de ces hypothèses explicatives ne signifie pas que nous considérons, pour notre part, que l'encouragement des citernes comme source d'eau potable représenterait une solution en soi au problème de la raréfaction des ressources d'eau potable et de leur renchérissement. Notre démarche est motivée par la question initiale (en partie question d'école) sur la place des dispositifs hors réseau dans les politiques du développement urbain durable. En tout état de cause, le développement des majel-s supposerait des enquêtes beaucoup plus approfondies et une politique de suivi sanitaire ( $c f$. encadré $n^{\circ} 2$ ).
Une première explication tiendrait au dédain que peut inspirer, aux yeux des tenants d'une culture technique moderne fondée sur le réseau, une solution traditionnelle et populaire, ou du moins perçue comme telle. Dans la même perspective, Treyer considère que les solutions de transferts interrégionaux d'eau ou encore la construction d'usines de dessalement, privilégiées par les ingénieurs de la SONEDE, relèguent à leurs yeux le potentiel des citernes dans l'ombre (Treyer, 2002, annexe 4, p. 39).

Nous proposons en complément de cette explication par les cultures techniques l'argument que les majel-s représentent potentiellement une menace économique pour l'économie du réseau. En effet, pour ses usagers, l'eau des majel-s apparaît comme gratuite. Ce faisant, ils négligent les coûts de construction du réservoir (nuls dans le cas des maisons anciennes) comme ses coûts d'entretien. Mais du point de vue de l'opérateur, l'eau des majel-s représente un manque à gagner d'autant plus important que les volumes consommés à partir de l'eau de pluie collectée viennent en déduction de la facture globale et, par construction, affectent particulièrement les recettes perçues sur les catégories des plus gros consommateurs. Leur impact financier renforce donc la fragilité structurelle des finances de l'entreprise et en particulier son mécanisme de péréquation interne. Inversement, de ce point de vue, encourager la construction de citernes pour les foyers ruraux qui de toute façon ont une faible consommation (en moyenne 60 l/habitant et par jour) est économiquement rationnel puisque ces consommateurs paient l'eau à un prix moyen inférieur au coût de revient moyen.

\section{CONCLUSION}

L'exemple de la distribution de l'eau potable en Tunisie présente un triple intérêt au regard de l'interrogation sur le développement urbain durable et l'organisation des services en réseau. Le premier élément consiste à souligner l'originalité du dispositif tunisien au regard des évolutions dans des pays voisins ou de contextes de pays émergents. Ayant fait de l'enjeu hydraulique un élément central de sa légitimité politique depuis l'indépendance, et désireux de conserver le contrôle sur l'accès aux services publics dans la période plus récente, le gouvernement a gardé la mainmise sur l'opérateur de la gestion de l'eau potable, au rebours des évolutions constatées notamment au Maroc, et plus largement dans nombre de pays comparables, où la gestion a été déléguée à des opérateurs privés. Mais la pérennisa- 
tion de ce système est passée par des évolutions dans la gestion interne pour la rendre plus performante. Cette maîtrise publique exigeante est sans doute l'un des facteurs qui expliquent la réussite dans I'application d'une politique rigoureuse de contrôle d'économie d'eau. Dans le contexte tunisien, le développement durable appliqué à l'eau potable se traduit principalement par l'impératif de l'économie de la ressource en eau.

L'outil tarifaire s'est avéré un élément central dans la mise en pratique de ce principe. Mais la politique suivie en matière de prix de l'eau est plus globale et articule deux autres éléments: l'équilibre financier de l'opérateur, dans un contexte de retrait de l'État de ses engagements économiques et sociaux; l'exigence d'intégration sociale et spatiale (urbain/rural) à travers la péréquation organisée par le tarif. Cet outil a eu de puissants effets, notamment en encourageant fortement l'adoption de pratiques de maîtrise de la consommation d'eau.

Comme constaté au Maroc (Tenesson, Rojat, 2003), certaines des évolutions liées à l'application de cette tarification semblent aller à l'encontre des objectifs de durabilité de la ressource: en particulier, la tendance de certains établissements industriels à se déconnecter du réseau se traduit par des pratiques illégales, non contrôlées et endommageant la ressource.

L'exemple des majel-s à Sfax apparaît très paradoxal. Cette pratique populaire ancienne mais remise au goût du jour témoigne d'une culture de l'économie d'eau qui, dans un contexte de gestion parcimonieuse de la ressource, ne peut qu'être bienvenue et devrait être, selon toute logique, encouragée. Toutefois, les premiers éléments d'analyse proposés ici dévoilent une situation plus complexe et laissent perplexe quant aux possibilités d'articulation entre le réseau et cette pratique hors réseau. En effet, les Sfaxiens recueillent l'eau de pluie pour la boire mais gaspillent l'eau du réseau public dont ils sont, par tête d'habitant, les plus gros consommateurs en Tunisie, notamment en arrosant leur jardin. D'autre part, alors que la situation financière de la compagnie gestionnaire de l'approvisionnement en eau potable est déjà tendue en conséquence de sa politique tarifaire et de ses obligations de service et de solidarité, l'impact économique du développement des majel-s ne peut que la fragiliser davantage.

Dans ce contexte, le manque apparent d'intérêt pour de telles pratiques, qu'explique sans doute aussi une puissante culture technique du réseau, n'a rien pour étonner. L'exemple tunisien est marqué par ce qu'on peut décrire comme l'idéal moderne du réseau universel au sommet de sa réussite, y compris comme instrument de mise en œuvre du développement durable. Dans cette situation, il n'y a guère de place pour les logiques du post-réseau.

Ali Bennasr, maître de conférence en Géographie à l'Université de Sfax, habilité à diriger les recherches, directeur adjoint du laboratoire Syfacte, est spécialiste de géographie industrielle et urbaine. II a aussi beaucoup travaillé sur l'aménagement et les mutations urbaines de la ville de Sfax et de sa région. Il s'intéresse aujourd'hui aux questions de développement durable à travers la thématique de l'étalement urbain et des inégalités environnementales. ali.bennasr@flshs.rnu.tn

Éric Verdeil est chercheur au CNRS, Université de Lyon, UMR Environnement Ville Société. Il a été pendant plusieurs années chercheur à I'Institut français du Proche-Orient à Beyrouth. II a notamment publié, avec G. Faour et $S$. Velut Atlas du Liban: territoires et société (éditions IFPO/CNRS Liban, 2007). Ses recherches portent sur les politiques urbaines au Moyen-Orient. eric.verdeil@normalesup.org

\section{NOTES}

(1) Cette contribution s'inscrit dans le cadre d'une collaboration de recherche bénéficiant d'une subvention du réseau Environnement et Développement Durable de I'Agence Universitaire de la Francophonie.

(2) À l'origine, les services d'eau potable étaient un simple département au sein du ministère de l'Agriculture.

(3) Où la desserte est assurée à $45 \%$ par le ministère de l'Agriculture.

(4) Le 22 mars est décrété journée nationale de l'économie d'eau. En 2008, cette journée a été célébrée sous le slogan " notre ressource en eau est rare, vitale et précieuse; tous ensemble pour l'économiser ».

(5) www.sonede.com.tn consulté le 31/3/2008.

(6) Le branchement individuel est encouragé à travers les facilités de paiement du compteur; de même que les prix qui augmentent en fonction des paliers incitent les abonnés à se procurer un compteur individuel, ce qui leur permet de contrôler leur consommation.

(7) En 2008, 1 Dinar tunisien équivaut à 0,6 Euro. Le Salaire Minimum Interprofessionnel Garanti (SMIG) est égal à 240 D/mois.

(8) Cette information est inscrite dans la page d'accueil du site de la SONEDE.

(9) « Sonede - Pourquoi tant d'égards à l'égard des "chers" abonnés? ", La Presse, 17décembre 2008.

(10) Toutefois, se référant à des données de 1999 ou 2000, Treyer (2002) fait état d'un taux de recouvrement de ses coûts par la SONEDE de l'ordre de 90\%, impliquant à cette date une 
subvention d'équilibre par l'État.

(11) On ne parle pas ici du secteur agricole mais uniquement des usagers industriels, touristiques ou domestiques gros consommateurs.

(12) Nasria en arabe signifie fontaines d'eau.

(13) Dans un exercice de prospective, Treyer envisage notamment la construction et/ou la réhabilitation de grandes citernes collectives, destinées à la collecte des eaux de pluie, en complément de la poursuite du développement des citernes dans les maisons individuelles.

(14) L'État, à travers les programmes de développement rural (1973-1992), a fourni des aides aux ruraux pour se doter de réservoirs.

\section{BibliographIE}

BANQUe mONDIale, 2003, Projet eau potable et assainissement. Rapport d'achèvement (PPD-37820; PDU-3782-A; PDU en DM-3782S; PPD-37830).

BENNASR A., 1993, La dynamique de l'industrie sfaxienne. Thèse de l'université. Faculté des Sciences humaines et sociales de Tunis.

BENNASR A., 2003, "L'étalement urbain de Sfax », Revue tunisienne de géographie, n³6, pp. 49-88.

BeNNASR A., 2004, "Les recompositions récentes de l'industrie sfaxienne ", Revue du centre d'étude et de recherches économiques et sociales, Tunis, $\mathrm{n}^{\circ} 130$.

CHABBI M., 1999, «La réhabilitation des quartiers populaires en Tunisie: de l'intégration à la régulation sociale », in Signoles P., Kadi G. el, Sidi Boumedine R. (dirs.), L'urbain dans le monde arabe. Politiques, instruments et acteurs, Paris, CNRS, pp. 187-200.

Communes du Grand Sfax, 2006, Stratégie de développement du Grand Sfax (2016), non publié.

Gana A., El Amrani M., 2006, «Crise hydraulique au Maghreb: raréfaction de la ressource ou problèmes de gestion », Géocarrefour, vol.81-1, pp. 37-50.

HAGGI A., 2006, "Présentation du secteur de l'eau potable. Bilans et perspectives", in Cinquante ans de développement humain au Maroc. Perspectives 2025. Rapport thématique Accès aux services de base, Cinquantenaire de l'Indépendance du Royaume du Maroc, pp. 59-77 (sur Internet: http://www.rdh50.ma)

Henia L. (sous la direction), 2008, Atlas de l'eau en Tunisie. Publication de la Faculté des Sciences Humaines et Sociales. Tunis

InStITUT MÉDITERRANÉEN DE L'EAU, 2002, Étude sur l'économie de I'eau chez le consommateur; Tunisie, Maroc, France, Espagne. (disponible sur Internet: http://www.ime-eau.org)

JAGLIN S. (coord.), 2004, "Services en réseau, services sans réseau dans les villes du Sud, dossier », Flux, n56-57.

JaGLIN S., 2005, Services d'eau en Afrique subsaharienne. La fragmentation urbaine en question, Paris, CNRS Éditions.

LeGros O., 2003, Le gouvernement des quartiers populaires. Production de l'espace et régulation politique dans les quartiers non-réglementaires de Dakar (Sénégal) et de Tunis, (Tunisie), Thèse de doctorat en géographie, Université de
Tours.

LimAM A., 2007, La tarification progressive, outil de gestion de la demande en eau: cas de l'eau potable en Tunisie, in Gestion de la demande en eau en Méditerranée, progrès et politiques, Plan Bleu, Zaragoza 19-21/03/2007

http://www.planbleu.org/publications/atelier_eau_saragoss e/EPI_5_TN_43_Limam_final_FR.pdf (le 1/7/2009)

Limam A., 2008, " L' eau potable rurale en Tunisie : Bilans et perspectives », Conférence 2nd MEDA Water Regional Event on Local Water Management, Marrakech. http://www.medawate-rmsu.org/meetings/SMWRE/Present ations/L\%E2\%80\%99EAU_POTABLE_RURALE_EN_TUNISI E.pdf.

MARIA A., 2008, "Bricolage ou innovation? Étude de systèmes alternatifs d'approvisionnement en eau dans les quartiers périphériques de Delhi », Idées pour le débat, n5 ("Entreprises et biens publics », Paris, IDDRI http://www.iddri.org)

Ministère DE L'AGRICULTURE, 2003, Stratégie à long terme du secteur de l'eau en Tunisie 2030. DGGR

Miras C. (DE), Le TelLieR J., Gouvernance urbaine et accès à l'eau potable au Maroc, Paris, L'Harmattan, 2005

PNUE - PLAN BLEU, Commission Méditerranéenne de Développement Durable, 2007, Stratégie Méditerranéenne pour le Développement Durable. Suivi des progrès dans le domaine de l'eau et promotion de politiques de gestion de la demande, rapport de la Tunisie.

RéPUBLIQUE TUNISIENNE, MINISTÈRE DE LA COOPÉRATION INTERNATIONale, World Bank, The Programme on Private Participation in Mediterranean Infrastructure, 2004, Study on the private participation in infrastructure in Tunisia, vol. 1

SONEDE, 2003, Étude de la tarification de l'eau potable. Idca consult, 2 rapports

SONEDE, 2007, Rapport annuel 2007, www.sonede.com.tn

TEnNeson M., Rojat D., 2003, "La tarification de I'eau au Maroc: comment servir différentes causes? », Politique africaine, $\mathrm{n}^{\circ} 205-1$, pp. 151-169.

Treyer S., 2002, Analyse des stratégies et prospectives de l'eau en Tunisie. T.I Monographie de l'eau en Tunisie. T.II Prospective de l'eau en Tunisie. Stratégie à long terme de la gestion de la demande en eau, Rapport pour le Plan Bleu, Sophia Antipolis. 